\title{
Cardiac arrest in the elderly with silent myocardial ischemia during general anesthesia
}

\author{
Soon Yul Kim, Woo Young Park, Jee Song Ghil, and Jae Chan Choi \\ Department of Anesthesiology and Pain Medicine, Wonju College of Medicine, Yonsei University, Wonju, Korea
}

Coronary artery disease (CAD) is a leading cause of mortality and morbidity in the elderly.

As with myocardial ischemia, some patients with myocardial infarct may be completely asymptomatic or symptoms may be so vague that they are unrecognized by the patient. Routine electrocardiogram (ECG) sometimes cannot diagnose the signs of it [1]. When we anesthetize the elderly with silent myocardial ischemia (SMI), he can be exposed to a high risk of cardiac arrest during anesthesia. If ECG cannot rule out SMI, We have to find another test for searching SMI. We experienced cardiac arrest of the elderly with SMI during general endotracheal anesthesia. We gave routine anesthesia to the elderly patient for ENT surgery, but after anesthesia, severe cardiac arrhythmia and arrest occurred. After proper treatment, though he recovered, he might have cardiovascular injuries. If one test checked out the elderly with SMI, the accident would not have occurred during anesthesia. We want to report the case and study with a literature review. A 65 years old man whose height and weight were $164 \mathrm{~cm}$ and $50 \mathrm{~kg}$, respectively, visited our hospital to undergo surgery for a laryngeal nodule under general endotracheal anesthesia. He did not present diabetes mellitus and hypertension in past history. The patient had a nodule-like opaque shadow of the right lung in chest PA, rightward axis and bradycardia (54 beats/ $\mathrm{min}$ ) in the electrocardiogram. The other laboratory findings were non-specific and he did not have any another symptoms. His blood pressure was 140/80 mmHg, pulse rate was 90 beats/ min, oxygen saturation of pulse oximeter was $97 \%$ on arrival of operation room. We induced the elderly gentleman with propofol $100 \mathrm{mg}$, atracurium $30 \mathrm{mg}$ after full mask ventilation with $100 \%$ oxygen. He was soon anesthetized, and intubated by direct laryngoscope with 6.o sized right angle preformed tube. When intubated, his blood pressure was 190/100 $\mathrm{mmHg}$, he was ventilated with a tidal volume of $500 \mathrm{ml}$ by an anesthetic machine. Continuous anesthetic maintenance was oxygen $2 \mathrm{~L} /$ min, N2O $2 \mathrm{~L} / \mathrm{min}$, sevoflurane $2-3$ vol\%. After 10 minutes, his cardiac rhythm was irregular in the electrocardiogram, blood pressure was not able to check in noninvasive blood pressure. Cardiopulmonary resuscitation was performed, He recovered and soon, vital signs were stable. The surgery was discontinued and he was transferred to a coronary angiographic room. We could see two coronary arteries stenosis, which were $80 \%$ stenosis in the circumflex branch of the left coronary artery and $50 \%$ stenosis in the middle branch of the right coronary artery in the coronary angiogram (Fig. 1). Aging is a universal and progressive physiologic process characterized by the declining end-organ reserve, decreased functional capacity, increasing imbalance of homeostatic mechanisms, and an increasing incidence of pathologic processes [2]. Especially in the cardiovascular system, the aging process is associated with primary and secondary changes in the heart, primary changes in the blood vessels, and alterations in autonomic control. As the heart ages, changes in morphology occur. Myocyte count deceases, left ventricular wall thickening occurs, and the conduction fiber density and sinus node cell number decrease [3]. Age related diseases in the cardiovascular system are hypertension, diabetes mellitus, ischemic heart diseases, and they exhibit a higher incidence in elderly individuals.

Corresponding author: Soon Yul Kim, M.D., Department of Anesthesiology and Pain Medicine, Wonju College of Medicine, Yonsei University, 162, Ilsan-dong, Wonju 220-701, Korea. Tel: 82-33-741-1536, Fax: 82-33-742-8198, E-mail: soonyul@yonsei.ac.kr

(c) This is an open-access article distributed under the terms of the Creative Commons Attribution Non-Commercial License (http:// creativecommons.org/licenses/by-nc/3.0/), which permits unrestricted non-commercial use, distribution, and reproduction in any medium, provided the original work is properly cited. 

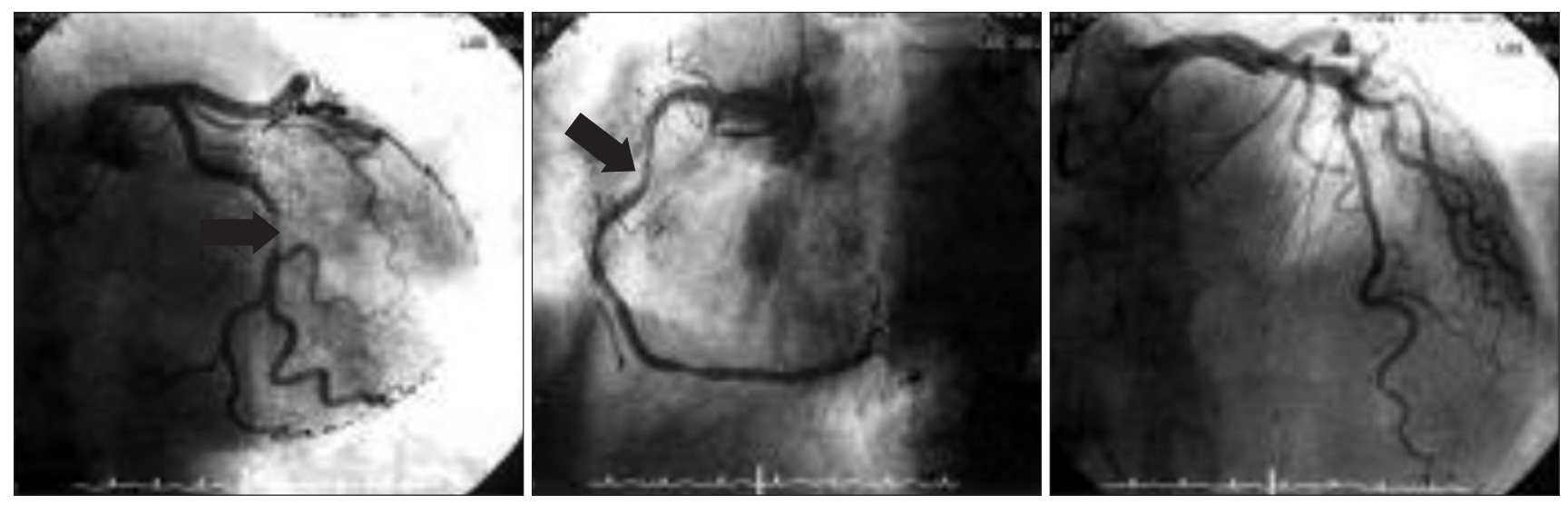

Fig. 1. This coronary angiography showed $80 \%$ stenosis on p-LCX coronary artery, $50 \%$ stenosis on m-RCA. It was checked on the first postoperative day. Black arrows indicate stenotic lesions of p-LCX coronary artery and m-RCA. p-LCX: proximal left circumflex, m-RCA: middle right coronary artery.

It is often difficult to separate the effects of aging from the effect caused by concurrent diseases. The elderly with DM, hypertension, peripheral arterial diseases, and family history have a higher possibility of ischemic heart disease. These factors become confusing when considering perioperative risk in elderly patients. SMI is defined as the objective evidence of myocardial ischemia in the absence of chest pain or other angina equivalents [4]. The reason for the frequent absence of chest pain in older patients with coronary arterial disease (CAD) is unclear because of the cognitive impairment with the inability to verbalize the sensation of pain, myocardial collateral circulation related to gradual progressive coronary arterial narrowing, and a reduced sensitivity to pain due to aging changes such as systemic or localized autonomic dysfunction [4]. The first manifestation of coronary artery disease in $60-$ $70 \%$ of patients is sudden death or myocardial infarction [5]. We need close monitoring for SMI and more aggressive treatment for the purpose of preventing catastrphpic events such as myocardial infarction or sudden death. The earlier identification of SMI patients results in the best prognosis.

\section{References}

1. Aronow WS. Silent MI. Prevalence and Prognosis in older patients diagnosed by routine electrocardiograms. Geriactric 2003; 58: 24-6, 36-8, 40 .

2. Weinert BT, Timiras PS. Invited review: Theories of aging. J Appl Physiol 2003; 95: 1706-16.

3. Priebe HJ. The aged cardiovascular risk patient. Br J Anaesth 2000; 85: 763-78.

4. Deedwania PC. Silent myocardial ischemia in the eldery. Drugs \& Aging 2000; 16: 381-9.

5. Oberman A, Kouchoukos NT, Holt JH Jr, Russell RO Jr. Long-term results of the medical treatment of coronary artery disease. Angiology 1977; 28: 160-8. 